\title{
Variation in the ICAM1-ICAM4-ICAM5 locus is associated with systemic lupus erythematosus susceptibility in multiple ancestries
}

\author{
Kwangwoo Kim, ${ }^{1}$ Elizabeth E Brown, ${ }^{2}$ Chan-Bum Choi, ${ }^{3}$ Marta E Alarc-n-Riquelme on \\ behalf of BIOLUPUS, ${ }^{4,5}$ Jennifer A Kelly, ${ }^{4}$ Stuart B Glenn, ${ }^{4}$ Joshua 0 Ojwang, ${ }^{4}$ Adam \\ Adler, ${ }^{4}$ Hye-Soon Lee, ${ }^{3}$ Susan A Boackle, ${ }^{6}$ Lindsey A Criswell, ${ }^{7}$ Graciela S Alarc- $n,{ }^{2}$ \\ Jeffrey C Edberg, ${ }^{2}$ Anne M Stevens, ${ }^{8}$ Chaim 0 Jacob, ${ }^{9}$ Gary S Gilkeson, ${ }^{10}$ Diane L \\ Kamen, ${ }^{10}$ Betty P Tsao, ${ }^{11}$ Juan-Manuel Anaya, ${ }^{12}$ Joel M Guthridge, ${ }^{4}$ Swapan K Nath, ${ }^{4}$ \\ Bruce Richardson, ${ }^{13}$ Amr H Sawalha, ${ }^{4,14,15}$ Young Mo Kang, ${ }^{16}$ Seung Cheol Shim, ${ }^{17}$ \\ Chang-Hee Suh, ${ }^{18}$ Soo-Kon Lee, ${ }^{19}$ Chang-sik Kim, ${ }^{20}$ Joan T Merrill, ${ }^{4}$ Michelle Petri, ${ }^{21}$ \\ Rosalind Ramsey-Goldman, ${ }^{22}$ Luis M Vil ,23 Timothy B Niewold, ${ }^{24}$ Javier Martin, ${ }^{25}$ \\ Bernardo A Pons-Estel, on behalf of GENLES ${ }^{26}$ Timothy J Vyse, ${ }^{27}$ Barry I Freedman, ${ }^{28}$ \\ Kathy L Moser, ${ }^{4}$ Patrick M Gaffney, ${ }^{4}$ Adrienne Williams, ${ }^{28}$ Mary Comeau, ${ }^{28}$ John \\ $\mathrm{D}$ Reveille, ${ }^{29}$ Judith A James, ${ }^{14} \mathrm{R}$ Hal Sco eld ${ }^{14}$ Carl D Langefeld, ${ }^{28}$ Kenneth M \\ Kaufman, ${ }^{4,15}$ John B Harley, ${ }^{30}$ Changwon Kang, ${ }^{1}$ Robert P Kimberly, ${ }^{2}$ Sang-Cheol Bae ${ }^{3}$
}

- Additional gures and tables are published online only. To view these les, please visit the journal online (http://ard.bmj.com/ content/early/recent)

For numbered af liations see end of article

\section{Correspondence to}

Changwon Kang, Korea Advanced Institute of Science and Technology, Daejeon 305-701, Republic of Korea; ckang@kaist.ac.kr or Robert P Kimberly, The University of Alabama at Birmingham, Birmingham, Alabama 35294, USA; Robert. Kimberly@ccc.uab.edu, or Sang-Cheol Bae, Hanyang University Hospital for Rheumatic Diseases, Seoul 133-792, Republic of Korea; scbae@hanyang.ac.kr

Received 30 November 2011 Accepted 24 February 2012 Published Online First 20 April 2012

$\mathrm{KK}$ and EEB contributed equally to this work and are joint rst authors. CK, RPK and SCB contributed equally to this work and are joint corresponding authors.

\section{ABSTRACT}

Objective Systemic lupus erythematosus (SLE; OMIM 152700) is a chronic autoimmune disease for which the aetiology includes genetic and environmental factors. ITGAM, integrin $\alpha_{M}$ (complement component 3 receptor 3 subunit) encoding a ligand for intracellular adhesion molecule (ICAM) proteins, is an established SLE susceptibility locus. This study aimed to evaluate the independent and joint effects of genetic variations in the genes that encode ITGAM and ICAM.

Methods The authors examined several markers in the ICAM1-ICAM4-ICAM5 locus on chromosome 19p13 and the single ITGAM polymorphism (rs1143679) using a large-scale case control study of 17481 unrelated participants from four ancestry populations. The singlemarker association and gene gene interaction were analysed for each ancestry, and a meta-analysis across the four ancestries was performed.

Results The A-allele of ICAM1-ICAM4-ICAM5 rs3093030, associated with elevated plasma levels of soluble ICAM1, and the A-allele of ITGAM rs1143679 showed the strongest association with increased SLE susceptibility in each of the ancestry populations and the trans-ancestry meta-analysis $\mathrm{CR}_{\text {meta }}=1.16,95 \% \mathrm{Cl} 1.11$ to $1.22 ; p=4.88 \times 10^{-10}$ and $\mathrm{OR}_{\text {meta }}=1.67,95 \% \mathrm{Cl} 1.55$ to $1.79 ; p=3.32 \times 10^{-46}$, respectively). The effect of the ICAM single-nucleotide polymorphisms (SNPS) was independent of the effect of the ITGAM SNP rs1143679, and carriers of both ICAM rs3093030-AA and ITGAM rs1143679-AA had an OR of 4.08 compared with those with no risk allele in either SNP $(95 \%$ Cl 2.09 to 7.98 ; $p=3.91 \times 10^{-5}$ ).

Conclusion These ndings are the rst to suggest that an ICAM integrin-mediated pathway contributes to susceptibility to SLE.
Systemic lupus erythematosus (SLE; OMIM 152700) is a chronic autoimmune disease for which the aetiology includes diverse genetic and environmental factors. The formation and deposition of immune complexes, which consist of nuclear autoantigens and autoantibodies, in vital organs result in chronic inflammation, organ failure and severe morbidity and mortality in patients with SLE.

Previous genome-wide and candidate-gene association studies reported an association of SLE with a non-synonymous variant in ITGAM, encoding integrin $\alpha_{M}$ (complement component 3 receptor 3 subunit), among European and African descents. ${ }^{1}$ ${ }^{2}$ ITGAM is a ligand for intercellular adhesion molecule (ICAM) proteins, suggesting the involvement of an integrin-ICAM-mediated adhesion pathway for SLE susceptibility.

In this candidate gene-association study, we examined the independent and combined effects of common variation in ITGAM and the ICAM1ICAM4-ICAM5 locus, encoding other components of the integrin-adhesion pathway, with SLE susceptibility using a large multi-ancestry population.

\section{METHODS}

\section{Study population}

As part of the first phase of this study, we included a total of 14719 patients with SLE and controls from diverse ancestry backgrounds, recruited from multiple institutions worldwide as part of the Lupus Association Study(LLAS)-2, with approval from the appropriate institutional review boards (online supplementary table S1). The affected had a minimum of four of eleven 1997 American College of Rheumatology revised criteria for the classification of SLE. ${ }^{3}$ All participants in this study were filtered 
by principal component analysis to identify population outliers as previously reported. ${ }^{4}$ The sample exceeding five SDs along any statistically significant principal component were defined as outliers and removed from the study.

For the second phase to demonstrate consistency of results for rs3093030 in this larger Korean population, we constituted an independent cohort consisting of 2762 SLE cases and controls of Korean ancestry. Hospital-based SLE cases and controls were recruited from six hospitals in Korea with approval from the respective institutional review boards (online supplementary table S1).

\section{Genotyping}

We genotyped the 12 ICAM variants in phase I (rs5030340, rs5030390, rs5030391, rs5030351, rs5491, rs1799969, rs5498, rs5030400, rs3093032, rs281437, rs3093030, rs2228615), which tagged most of the common single-nucleotide polymorphisms (SNPs) in the ICAM1-ICAM4-ICAM5 locus, in addition to an ITGAM variant (rs1143679), using customised arrays based on the Illumina iSelect platform at the Lupus Genetics Studies Unit of the Oklahoma Medical Research Foundation. For the SLEassociated SNP in the ICAM1-ICAM4-ICAM5 locus with the strongest effect (rs3093030), we genotyped this marker for the phase II Korean cohort using the Sequenom iPlex platform at the Korea Advanced Institute of Science and Technology.

\section{SNP association with SLE susceptibility}

The statistical assessment for SNP association with SLE susceptibility was performed by the $\chi^{2}$ test with allelic genetic model for each ancestry and meta-analysis across the four ancestries using the PLINK 1.07 (http://pngu.mgh.harvard.edu/ purcell/ plink/). In the meta-analysis, homogeneity of effect sizes was evaluated using the $I^{2}$ heterogeneity index and Cochrane's $Q$ statistic. ${ }^{6}$ For $p>0.01$ in heterogeneity $Q$ statistic, fixed-effects models were implemented; otherwise random-effects models were used. For meta-analysis, GWAMA 1.4 software (http:// www.well.ox.ac.uk/gwama/) was also used to calculate OR and $95 \% \mathrm{CI}^{7}$ Hardy-Weinberg equilibrium (HWE) was examined by comparison between genotype distributions expected and observed in controls using the $\chi^{2}$ test. Pair-wise $\mathrm{D}^{\prime}$ and $r^{2}$ values between SNPs were calculated by the Haploview 4.2 program. Statistical power was estimated using the CaTSPower calculator (http://www.sph.umich.edu/csg/abecasis/ CaTS/index.html).

\section{Imputation}

Using the genotype data for the 12 ICAM SNPs, 58 HapMap SNPs located in a chromosome 19 region from position $10200 \mathrm{~kb}$ to $10320 \mathrm{~kb}$ (build 36.3) were imputed using the MACH V.1.0 program (http://www.sph.umich.edu/csg/abecasis/MACH/index. html) with the ancestry-matched template data provided by the International HapMap Project (http://www.hapmap.org) Phase 3 (Genome Browser release 2). Europeans were imputed using the CEU (Utah residents with Northern and Western European ancestry) data, Africans using the ASW (African ancestry in Southwest USA) data, Hispanics using the MEX (Mexican ancestry in Los Angeles, California) data, and Koreans using the CHB (Han Chinese in Beijing, China) data. Among the 58 imputed SNPs, $69.0 \%$ in Europeans, $77.6 \%$ in Africans, $58.6 \%$ in Hispanics and $63.8 \%$ in Koreans showed high $(\geq 0.70)$ average posterior probability for the most likely genotype, although some SNPs were not confidently imputed because of their weak correlations with the 12 genotyped SNPs.

\section{Gene-gene interaction}

Epistatic interactions between the seven SLE-associated SNPs in the ICAM1-ICAM4-ICAM5 locus and the non-synonymous SNP rs1143679 in ITGAM were examined using multiplicative logistic regression with the affected status as a dependent factor, two SNPs as main factors and the two-SNP interaction term. The analysis was performed using the PLINK '--epistasis' option. ${ }^{5} 8$ In addition, case-only analysis using the PLINK '--fast-epistasis' and '--case-only' options was used to examine the dependency or the correlation between the tested SNPs in the groups of SLE cases. ${ }^{5}$ To access combined effects of the risk alleles in rs3093030 and rs1143679, fixed-effects OR and 95\% CI were estimated in stratification by the number of risk alleles (0 to 4 ) as compared with the group having no risk allele, separately among the European, African and Hispanic populations using the Case-control And Tdt Meta-Analysis Package (http:// cran.r-project.org/web/packages/catmap/index.html). Twocopy carriers homozygous for ICAM or ITGAM risk allele were removed from the two-risk-allele carrier group in order to see the combined effect.

\section{SNP association with gene expression}

Using the Genevar 2.0.1 database, which includes SNP genotype and mRNA profiling data for fibroblasts, lymphoblastoid cell lines, and T cells from the 75 GenCord individuals, ${ }^{9} 10$ the 19 SNPs located in a chromosome 19 region from position $10200 \mathrm{~kb}$ to $10320 \mathrm{~kb}$ were assessed for their associations with mRNA levels of ICAM1, ICAM4 and ICAMS using a $t$ statistic for Spearman's rank correlation coefficient $(\rho)$. To minimise the type I error caused by multiple testing, the significance level of the $\mathrm{p}$ value was set to $\alpha=0.05 /(19 \mathrm{SNPs}) /($ five probes)/(three cell lines) $=1.75 \times 10^{-4}$

\section{RESULTS \\ ICAM-SLE association study}

In phase I, we evaluated 12 common variants directly typed in the ICAM1-ICAM4-ICAM5 locus spanning $22 \mathrm{~kb}$ in a total of 14719 unrelated SLE cases and controls of European ( $\mathrm{n}=7427$; SLE/controls=3936/3491), African ( $\mathrm{n}=3613 ; 1679 / 1934)$, Hispanic $(\mathrm{n}=2299$; 1492/807) and Korean ( $n=1380 ; 640 / 740)$ ancestries (online supplementary table S1). Proportions of genetic ancestry for the study subjects were filtered using principal component analysis as described in a previous study (online supplementary figure S1). ${ }^{4}$ All variants were typed with call rates of over $97.9 \%$, and genotype proportions were consistent with expectations of the HWE $(p \geq 0.001)$ among controls from each of the four ancestries (online supplementary table S2).

Of the 12 ICAM SNPs typed, five were significantly associated with SLE susceptibility under a statistical threshold of $\mathrm{p} \leq 4.17 \times 10^{-3}$ in the trans-ancestry meta-analysis after Bonferroni correction for multiple comparisons (rs5030390, rs5498, rs281437, rs3093030 and rs2228615; $1.05 \times 10^{-8} \leq \mathrm{p}_{\text {meta }} \leq 2.80 \times 10^{-4}$ ) (online supplementary table S2). Moreover, the directions of the effect sizes of each SNP were same among the ancestries except for rs5030390, the association of which was considered unreliable (online supplementary table S2).

The strongest association with SLE was found with rs3093030 located in the $0.4 \mathrm{~kb}$ intergenic region between ICAM1 and ICAM4 $\left(\mathrm{p}=2.49 \times 10^{-8} ; \mathrm{OR}=1.16,95 \%\right.$ CI 1.10 to 1.22$)$. The effect size was similar in each of the four ancestries (online supplementary table S2), with $\mathrm{OR}=1.12$ (95\% CI 1.05 to 1.20$)$ in European, $\mathrm{OR}=1.18$ (95\% CI 1.01 to 1.37 ) in African, $\mathrm{OR}=1.28$ (95\% CI 1.13 to 1.45 ) in Hispanic and $\mathrm{OR}=1.15$ (95\% CI 0.98 
Table 1 Association of directly typed and imputed markers in the ICAM locus and ITGAM with susceptibility to systemic lupus erythematosus

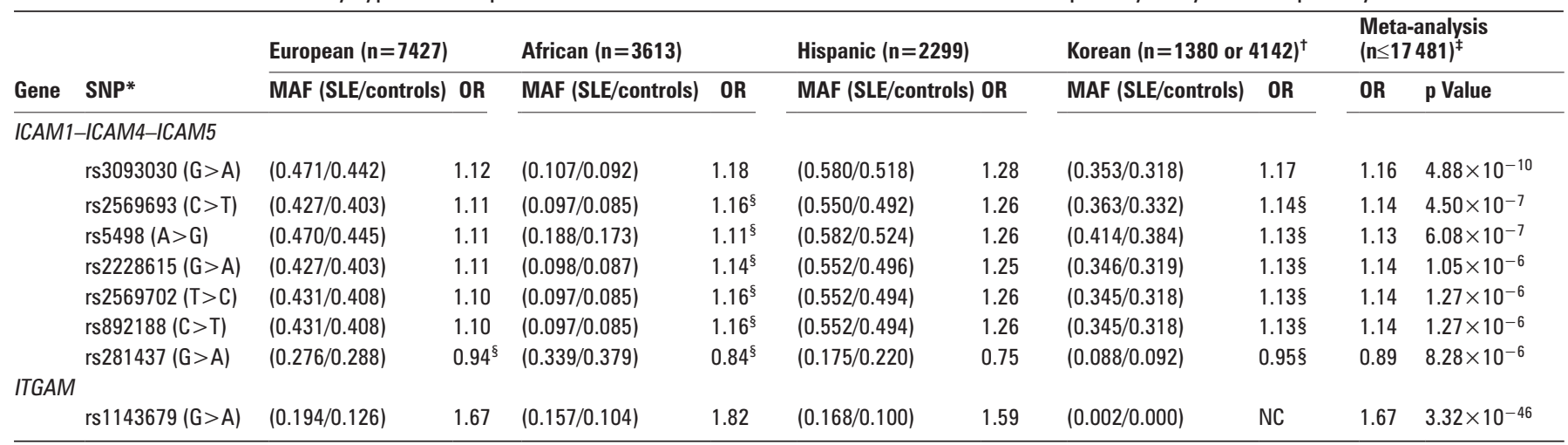

${ }^{*}$ Four SNPs (rs3093030, rs5498, rs2228615 and rs281437) were genotyped and the other three SNPs (rs2569693, rs2569702 and rs892188) were imputed. The major allele is more common than the minor allele in the whole control population but not necessarily in each subpopulation.

${ }^{\dagger} \mathrm{A}$ total of 4142 Korean participants from phase I and phase II were genotyped for rs3093030, and the separate analyses are shown in online supplementary table S2.

${ }^{\ddagger}$ Fixed-effects $p$ value and OR were calculated from the meta-analysis because the effect size of each SNP was not significantly different among the ancestry populations ( $p>0.01$ in

Cochran's 0 statistic). For ITGAM rs1143679, the meta-analysis was performed with European, African and Hispanic results.

${ }^{\S}$ Allelic associations with SLE were not statistically significant at a threshold of $\alpha=0.05$. The detailed information is shown in online supplementary table S2.

MAF, minor allele frequency; NC, not calculated; SLE, systemic lupus erythematosus; SNP, single-nucleotide polymorphism.

to 1.35$)$ in Korean ancestries $\left(\mathrm{p}=0.307\right.$ in $\mathrm{Q}$ statistic, $\left.I^{2}=17.0\right)$. In a conditional logistic regression analysis followed by a metaanalysis, we found that the SLE association of rs5498, rs281437 and rs2228615 accounted for that of rs3093030, and they presented a consensus signal of SLE susceptibility locus, possibly because they are in high linkage disequilibrium with rs3093030 (D' $\geq 0.84$; online supplementary table S3).

Although the effect magnitude of rs3093030 among Koreans was similar to those of the other ancestry populations, it did not achieve statistical significance, in contrast with the others $\left(p=0.0841\right.$ in Koreans versus $p=5.95 \times 10^{-4}$ in Europeans, $\mathrm{p}=4.02 \times 10^{-2}$ in Africans and $\mathrm{p}=7.28 \times 10^{-5}$ in Hispanics), perhaps because of the small sample size of the Korean population relative to the other ancestry populations. With the minor allele frequency of 0.324 in the Korean controls, the statistical power to detect the effect size found in the meta-analysis (fixed-effects $\mathrm{OR}=1.16$ ) was only $42 \%$ in the Korean population.

To increase the statistical power for Korean detection, in phase II we recruited an additional independent population of 2762 unrelated Korean participants (online supplementary table S1). Genotyping call rate was $96.9 \%$, and the control subject genotypes were distributed under HWE ( $p=0.691)$. Indeed, a similar effect size was detected in the validation population $(\mathrm{OR}=1.18)$, and by combining the phase I and phase II populations of Koreans $(n=4142)$, statistical significance $\left(\mathrm{p}=1.07 \times 10^{-3} ; \mathrm{OR}=1.17,95 \%\right.$ CI 1.07 to 1.29$)$ was achieved (online supplementary table S2). Therefore, with the additional Korean validation population, each of the four ancestry populations independently showed a statistically significant association between the ICAM locus SNP rs3093030 and SLE susceptibility, and the meta-analysis across the four ancestral populations revealed a more significant overall relationship $\left(p=4.88 \times 10^{-10}\right.$; $\mathrm{OR}=1.16,95 \%$ CI 1.11 to 1.22 ; online supplementary table S1).

To further characterise this genomic region as an SLE susceptibility locus, we imputed HapMap SNPs around the ICAM gene cluster that included EDG5, MRPL4, ICAM1, ICAM4, ICAM5, RAVER1 and ICAM3. Using the genotype data for the 12 ICAM SNPs, we imputed 58 HapMap SNPs located in a chromosome 19 region from position $10200 \mathrm{~kb}$ to $10320 \mathrm{~kb}$ (Genome build 36.3) using the MACH V.1.0 program, with the ancestry-matched haplotype templates provided by the HapMap3 Genome Browser release 2 of the International HapMap Project.
Three imputed SNPs (rs2569693, rs2569702 and rs892188) with high imputation quality (average posterior probability for the most likely genotype $\geq 0.97$ ) were significantly associated with SLE susceptibility (table 1) while demonstrating the same direction of SLE-risk effects in each of the SNPs. In addition, all SLE-associated SNPs showed larger effects in Hispanics than in the other ethnic groups. These imputed SLE-associated SNPs were localised to the ICAM1-ICAM4-ICAM5 region and correlated highly with the genotyped SLE-associated SNP rs3093030 $\left(0.81 \leq r^{2} \leq 0.96\right)$. Trans-ancestry comparisons for the seven SLEassociated SNPs (ie, four genotyped and three imputed) supported that the ICAM1-ICAM4-ICAM5 locus alone associated sufficiently with SLE susceptibility ( $\mathrm{p} \leq 8.28 \times 10^{-6}$; figure 1$)$.

\section{Pathway-based ICAM and ITGAM interaction study}

Because ICAM 1 and ICAM4 are binding partners of an $\alpha_{M} \beta_{2}$ integrin, in which the $\alpha$ subunit is encoded by an SLE-susceptibility gene, ITGAM, we examined the statistical interaction between SLE-risk alleles of the ICAM1-ICAM4-ICAM5 region and the previously reported SLE-risk alleles of the ITGAM SNP rs1143679 to characterise the pathway-based effect on SLE susceptibility. ${ }^{2}$ For this analysis, all participants of the phase I population were also genotyped for the non-synonymous SNP rs1143679 located in exon 3 of ITGAM. Call rates were $\geq 99.6 \%$ within each ancestry population, and genotype distributions were consistent with expectations of HWE in each of the control groups (online supplementary table S2).

The overall fixed effect of the SLE-risk-associated A allele in the ITGAM SNP was OR=1.67 (95\% CI 1.55 to $\left.1.79, \mathrm{p}=3.32 \times 10^{-46}\right)$, showing similar effect sizes among European, African and Hispanic ancestries ( $p=0.541$ in $\mathrm{Q}$-statistic, $I^{2}=0.00 \%$; online supplementary table $\mathrm{S} 2$ ), although the risk-associated allele was too rare $(0.07 \%)$ to evaluate the interaction among Koreans. The infrequency in Koreans was consistent with the previous report. ${ }^{11}$ Statistical interaction between ITGAM rs1143679-A and each of the seven SLE-associated SNPs in the ICAM1-ICAM4-ICAMS locus $\left(\mathrm{p} \leq 4.17 \times 10^{-3}\right.$; table 1$)$ were examined among cases and controls using the PLINK '-- epistasis' analysis and among only the cases using the PLINK '--fast-epistasis' analysis.

The ITGAM and ICAM SNPs independently affected SLE susceptibility, showing no notable ICAM-ITGAM epistatic interactions ( $p>0.05$; online supplementary table S4). Using five 


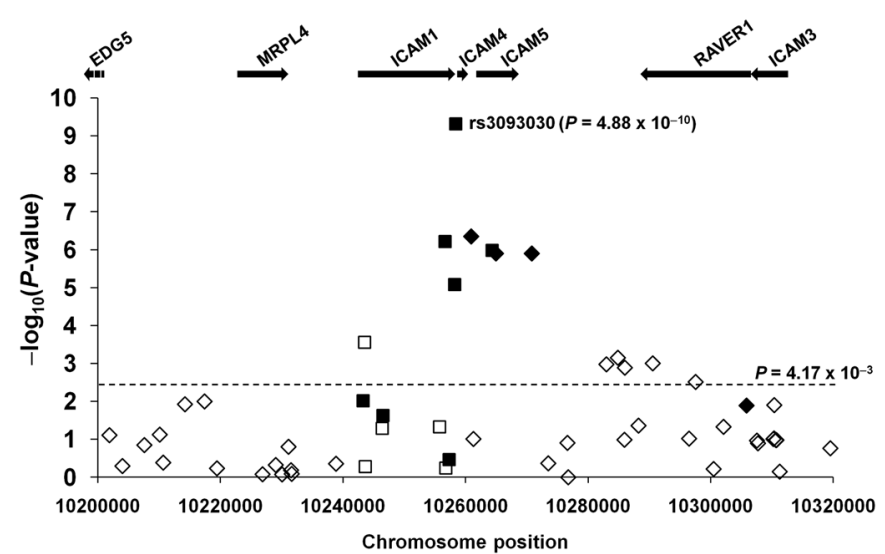

Figure 1 A systemic lupus erythematosus association map for the genotyped (squares) and imputed single-nucleotide polymorphisms (SNPs) (diamonds) within and around the ICAM1-ICAM4-ICAM5 locus. Negative logarithms (base 10) of the $p$ values calculated from the meta-analysis (y axis) are plotted against SNP positions on human chromosome 19 ( $x$ axis). The direction of effect size was the same in all four ancestries for some SNPs (filled symbols) but not for others (empty symbols). The arrows indicate transcription directions and gene sizes.

subgroups according to the number of risk-associated alleles (0 to 4 ) in the ICAM SNP, rs3093030, and the ITGAM SNP, rs1143679, we assessed the combined effects of the SLE risk-associated alleles in the ICAM-integrin pathway. Fixed-effects $O R$ and 95\% CI for SLE susceptibility in each subgroup were estimated with reference to the subgroup with no risk allele. The natural logarithm of $\mathrm{OR}$ correlated linearly $\left(r^{2}=0.9803\right)$ with the number of risk alleles (0 to 4), and four-copy carriers had OR of 4.08 for risk of SLE compared with carriers with 0 copies (figure 2).

\section{Gene expression}

To determine whether the genetic variation has a role in gene expression, we evaluated expression quantitative trait loci (eQTL) associations within the ICAM locus using the Genevar (GENe Expression VARiation) database (http://www.sanger. ac.uk/resources/software/genevar/), which publicly provides data for 75 GenCord individuals on both SNP genotypes and expression profiles from primary fibroblasts, lymphoblastoid cell lines and T cells. ${ }^{9} 10$ The 19 Genevar SNPs included rs2569693, rs5498, rs2228615 and rs281437 among the SLEassociated SNPs. However, none of the evaluated markers were associated with aberrant mRNA levels of ICAM1, ICAM4 or ICAMS in any cell type (online supplementary figure S2).

\section{DISCUSSION}

To our knowledge, this is the first report of a comprehensive approach used to evaluate the effect of the ICAM1-ICAM4ICAMS locus on SLE risk. We observed that several SNPs within this locus are associated with susceptibility to SLE in multiple ancestry populations.

ICAM1 is mainly expressed in the vascular endothelium, macrophages and lymphocytes, and plays a role in immunological events including extravasation and T-cell-mediated responses. ${ }^{12}$ However, a plausible role for ICAM4 and ICAM5 in SLE pathogenesis is less clear because they are preferentially expressed in red blood cells and brain, respectively. ${ }^{13} 14$

The ICAM polymorphisms were not associated with altered mRNA levels of ICAM1, ICAM4 or ICAMS in the Genevar database, although the sample size $(n=75)$ was too small to make this null association conclusive. However, soluble ICAM1 levels

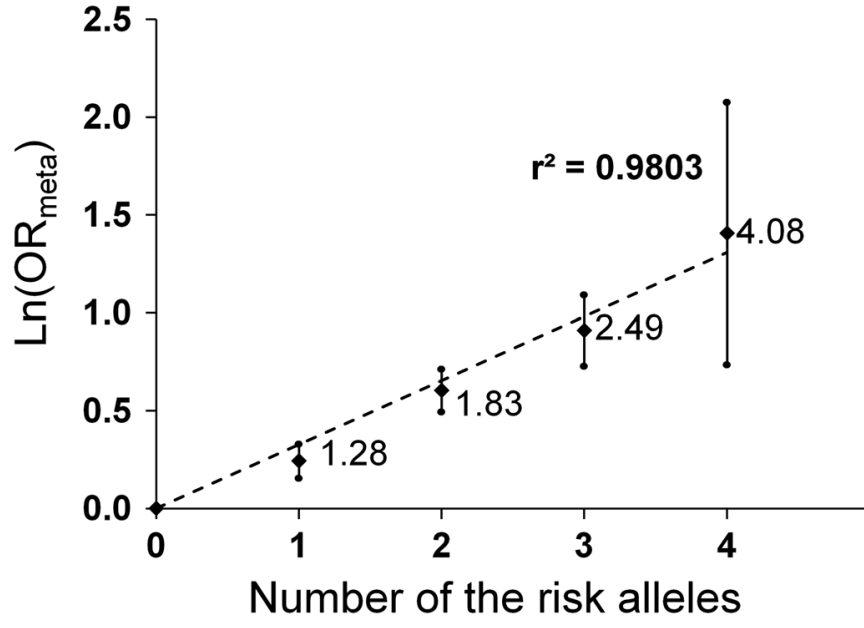

Figure 2 Combined effects of ICAM1-ICAM4-ICAM5 rs3093030-A and ITGAM rs1143679-A alleles on systemic lupus erythematosus (SLE) susceptibility. Natural logarithms of the relative odds of SLE (y axis) are plotted against the number of at-risk alleles ( $x$ axis) in the two putative SLE susceptibility single-nucleotide polymorphisms (SNPs), rs3093030 in ICAM1-ICAM4-ICAM5 and rs1143679 in ITGAM. OR values are provided next to the data points, and error bars represent corresponding $95 \% \mathrm{Cl}$. A linear trend line (dashed) and $r^{2}$ are shown.

in plasma have been associated with two SNPs (rs3093030 and rs5498) in the ICAM1-ICAM4-ICAM5 locus by protein QTL analysis in two independent studies involving 6578 European women $\left(p=5.9 \times 10^{-23}\right.$ and $\left.p=4.8 \times 10^{-25}\right)$ and 9813 European individuals $\left(p=3.5 \times 10^{-23}\right.$ and $\left.p=2.5 \times 10^{-21}\right) .{ }^{15} 16$ The alleles that were associated with increased soluble ICAM1 in those studies (A in rs3093030 and G in rs5498) were also associated with increased susceptibility to SLE in this study, providing a plausible mechanism for their effect. In addition, the genotype-phenotype relationships are consistent with several previously reported observations of raised plasma levels of soluble ICAM1 in patients with SLE, ${ }^{17-21}$ suggesting an underlying aetiological role.

Soluble ICAM1 is a cleaved form consisting of the extracellular domain of the membrane-bound full-length ICAM1 that is capable of binding to ligands. Thus soluble and membrane-bound ICAM1 can compete with each other for binding to ligands, ${ }^{12}$ but the immunological role of either soluble or membrane-bound ICAM1 in SLE pathogenesis has not been characterised. The level of soluble ICAM1 probably correlates with that of membrane-bound ICAM1,12 19 but it is not known whether the increase of soluble ICAM1 in SLE accompanies with the concurrent increase of the membrane form due to increased gene expression or with the reciprocal decrease of the membrane form due to increased proteolytic processing, as the membrane-bound ICAM1 levels have not been measured in patients with SLE. None of the Genevar SNPs in the ICAM1-ICAM4-ICAM5 locus was associated with ICAM1 mRNA expression. Instead, the non-synonymous ICAM1 SNP rs5498 (encoding Lys469Glu) was associated with both increased levels of the soluble form of ICAM1 protein and increased SLE susceptibility. Of note is the fact that it is located at an Ig superfamily domain, which may affect the proteolytic conversion to the soluble ICAM1. However, the level of statistical significance for the SLE association of rs5498 was weaker than that of rs3093030 in all populations, and statistical significance was not reached in the African or Korean ancestry populations. Thus it is possible that rs5498 affects SLE susceptibility in some ancestry populations but not others. 
In this study, we also showed the association of ITGAM rs1143679 in European, African and Hispanic descendants, which was also found in previous association studies for systemic sclerosis. ${ }^{22}$ This common variant was monomorphic in Koreans, which is consistent with the literature of other Asian populations, ${ }^{11}$ perhaps reflecting selective pressures in this population. ITGAM (also known as CD11b, Mac-1 and complement receptor type 3 ) is a well-characterised molecule in the integrin $\alpha$ chain family that is expressed in a number of myeloid cells including macrophages, monocytes and neutrophils. ${ }^{23-25}$ ITGAM encodes the $\alpha$ chain of $\alpha_{M} \beta_{2}$ integrin, which regulates neutrophil and monocyte adhesion and migration from the bloodstream via interactions with a wide range of structurally unrelated ligands, including but not limited to ICAM1 and ICAM2. ${ }^{2627}$

The previously identified SLE-susceptibility ITGAM SNP, rs1143679, encodes a change in amino acid from arginine at position 77 to histidine $(\mathrm{R} 77 \mathrm{H})$. This conversion of amino acids induces an alteration in the tertiary and quaternary structures of the ligand-binding $\alpha_{M} \beta_{2}$ domain, thereby modifying its overall binding affinity. ${ }^{28}$ Nath et al suggest that the $\mathrm{R} 77 \mathrm{H}$ conversion may ultimately influence the conformation of the $\alpha I$ domain (specific to the ligand-binding domain for ICAM1), with subsequent consequences on $\alpha_{M} \beta_{2}$ ligand binding. ${ }^{2}$

Indeed, evidence suggests that $\alpha_{M} \beta_{2}$ levels are increased on neutrophils in SLE patients with active disease and may contribute to endothelial injury, consistent with the cumulative organ damage associated with SLE. ${ }^{29} \alpha_{M} \beta_{2}$ is also involved in immune complex clearance, a process that is impaired in patients with SLE, ${ }^{30}$ suggesting that the function of $\alpha_{M} \beta_{2}$ may be altered in these people, either directly by structural modification or indirectly by alterations in receptor-ligand binding, possibly resulting from structural modification. ${ }^{2}$

In addition, this conversion is a target for alloantibodies present in mothers of neonates affected with neonatal autoimmune neutropenia. ${ }^{28}$ Notably, alloantibodies reactive against the polymorphic $\alpha_{M} \beta_{2}$ molecule are capable of blocking the $\alpha_{M} \beta_{2}$ dependent adhesion of neutrophils and monocytic U937 cells to a number of molecules including ICAM1. ${ }^{28}$

The functional association of ICAM and ITGAM prompted us to examine the associated SNPs in these genes for epistatic effects. Although a joint role for both ICAM1 and ITGAM in SLE aetiology appears to be plausible, they independently affected SLE susceptibility in this study. This may indicate that the functional role of the ICAM variants is involved in a nonintegrin-mediated pathway or that some factors are affected by ICAM-ITGAM binding through different pathways of ICAM and ITGAM without a common pathway.

In summary, we identified the association of several SNPs within the ICAM1-ICAM4-ICAM5 locus with SLE susceptibility. Although the effect size was modest, the association was consistent across all four ancestries. Our findings suggest that common variations in genes involved in ICAM-mediated or ICAM/integrin-mediated adhesion play a causal role in SLE susceptibility.

Contributors KK, EEB, JAK, J00, SKN, BR, AHS, KLM, PMG, JBH, CK, RPK and S-CB designed the study. EEB, C-BC, MEA-R, H-SL, SAB, LAC, GSA, JCE, AMS, COJ, GSG, DLK, BPT, J-MA, JMG, YMK, SCS, C-HS, S-KL, C-SK, JTM, MP, RRG, LMV, TBN, JM, BAP-E, TJV, BIF, KLM, PMG, JDR, JAJ, RHS, JBH, RPK and S-CB recruited and characterised the SLE cases and controls. KK, AA and KMK performed genotyping. SBG, AW, MC and CDL performed quality control analyses. KK and EEB performed statistical analyses. KK, EEB, C-BC, CK, RPK and S-CB prepared the manuscript. All authors approved the nal draft.

Acknowledgements We thank the study participants and physicians who provided samples (Peter K Gregersen, So-Young Bang, BIOLUPUS Network and GENLES Network) and technical support (Taehyeung Kim). The members of the BIOLUPUS Network who provided samples used in this study are Sandra D Alfonso, Rafaella
Scorza, Mauro Galleazzi, Gian Domenico Sebastiani, MD Danieli, and Sergio Migliaresi in Italy, Bernard R Lauwerys in Belgium, Emoke Endreffy and L szl-Kov cs in Hungary, Carlos Vasconcelos and Berta Martins da Silva in Portugal, L-igo R a Figueroa, Carmen Gutierrez, Ana Su rez, Norberto Ortego, Jos Luis Calleja, Enrique de Ram-nGarrido, Julio Sanchez Rom n, and Mario Sabio in Spain, Helle Laustrup and Peter Junker in Denmark, and Marc Bijl and Cees Kallenberg in Holland; Dr Lennart Truedsson provided controls from Southern Sweden (Lund). The members of the GENLES Network who provided samples used in this study are Hugo R Scherbarth, Pilar C Marino, Estela L Motta, Susana Gamron, Cristina Drenkard, Emilia Menso, Alberto Allievi, Guillermo A Tate, Jose L Presas, Simon A Palatnik, Marcelo Abdala, Mariela Bearzotti, Alejandro Alvarellos, Francisco Caeiro, Ana Bertoli, Sergio Paira, Susana Roverano, Cesar E Graf, Estela Bertero, Cesar Caprarulo, Griselda Buchanan, Carolina Guiller- $\mathrm{n}$, Sebastian Grimaudo, Jorge Manni, Luis J Catoggio, Enrique R Soriano, Carlos D Santos, Cristina Prigione, Fernando A Ramos, Sandra M Navarro, Guillermo A Berbotto, Marisa Jorfen, Elisa J Romero, Mercedes A Garcia, Juan C Marcos, Ana I Marcos, Carlos E Perandones, Alicia Eimon and Cristina G Battagliotti in Argentina; Eduardo Acevedo and Mariano Cucho in Per ; Ignacio Garc'a de la Torre, Mario Cardiel R'os, Jos Francisco Moctezuma and Marco Maradiaga Cece-a in Mexico.

Funding The work was supported by grants from US NIH (Al063622, Al071651, Al082714, Al083194, Al094377, Al24717, AR042460, AR043274, AR043814, AR044804, AR048940, AR052300, AR053483, AR058554, AR060366, AR43727, AR62277, CA141700-01, GM063483, HD07463, K08-Al083790, K24-AR002138, M01-RR00079, N01-AR-6-227, P01-AR49084, P30-DK42086, P30-AR055385, P60-AR049459 P60-AR053308, P60-2-AR30692, P01-AR49084, PR094002, R01-AR33062, R21-Al070304, RR015577, RR020143, UL1RR024999, UL1RR025005, UL1RR025741, UL1RR029882 and 5UL1RR025777), the Lupus Foundation of America, the Alliance for Lupus Research, a Kirkland Scholar Award, the US Department of Veterans Affairs, the Korean Healthcare Technology Research and Development Project (A111218-11-GM01), the Korea Research Program for New Drug Target Discovery (20090083335), the National Research Foundation of Korea (2010-0014162), the Lupus Research Institute Novel Research Grant, the Alliance for Lupus Research Target Identi cation in Lupus Grant, the Arthritis National Research Foundation Eng Tan Scholar Award, the ESF in the framework of the Research Networking Programme European Science Foundation The Identi cation of Novel Genes and Biomarkers for Systemic Lupus Erythematosus (BIOLUPUS) 07-RNP-083, the Swedish Research Council, the Swedish Association against Rheumatism, the Swedish International Development Agency, the Gustaf Vth 80th-Jubilee Foundation, the Instituto de Salud Carlos III (PS09/00129) partly nanced by the FEDER funds of the European Union, and a grant from the Consejer'a de Salud de la Junta de Andaluc'a.

Competing interests None.

Patient consent Obtained.

Ethics approval The study was approved by the Institutional Review Board of all participating institutions.

Provenance and peer review Not commissioned; externally peer reviewed.

Author affiliations ${ }^{1}$ Department of Biological Sciences, Korea Advanced Institute of Science and Technology, Daejeon, Korea

2Departments of Medicine and Epidemiology, Schools of Medicine and Public Health,

The University of Alabama at Birmingham, Birmingham, Alabama, USA

${ }^{3}$ Department of Rheumatology, Hanyang University Hospital for Rheumatic Diseases,

Seoul, Korea

${ }^{4}$ Arthritis and Clinical Immunology Program, Oklahoma Medical Research Foundation, Oklahoma City, Oklahoma, USA

${ }^{5}$ Area of Human DNA Variability, Centro de Gen-mica e Investigaci-n Oncol-gica

(GENYO), P zer-Universidad de Granada-Junta de Andaluc'a, Granada, Spain

${ }^{6}$ Division of Rheumatology, University of Colorado Denver School of Medicine, Aurora, Colorado, USA

${ }^{7}$ Rosalind Russell Medical Research Center for Arthritis, University of California, San Francisco, California, USA

${ }^{8}$ University of Washington, Seattle Children s Hospital, Seattle, Washington, USA

${ }^{9}$ The Lupus Genetic Group, University of Southern California, Los Angeles, California, USA

${ }^{10}$ Department of Medicine, Medical University of South Carolina, Charleston, South Carolina, USA

${ }^{11}$ David Geffen School of Medicine, University of California, Los Angeles, California, USA ${ }^{12}$ Center for Autoimmune Diseases Research, Universidad del Rosario, Bogota,

Colombia

${ }^{13}$ Division of Rheumatology, University of Michigan and US Department of Veterans

Affairs Medical Center, Ann Arbor, Michigan, USA

${ }^{14}$ Department of Medicine, University of Oklahoma Health Sciences Center, Oklahoma City, Oklahoma, USA

${ }^{15}$ Department of Medicine, US Department of Veterans Affairs Medical Center, Oklahoma City, Oklahoma, USA

${ }^{16}$ Department of Internal Medicine (Rheumatology), Kyungpook National University School of Medicine, Daegu, Korea 
${ }^{17}$ Division of Rheumatology, Department of Medicine, Eulji Medi-Bio Research Institute, Eulji University, Daejeon, Korea

${ }^{18}$ Department of Rheumatology, Ajou University School of Medicine, Suwon, Korea

${ }^{19}$ Department of Internal Medicine, Yonsei University College of Medicine, Seoul, Korea

${ }^{20}$ Department of Ophthalmology, Chungnam National University School of Medicine, Daejeon, Korea

${ }^{21}$ Department of Medicine, Johns Hopkins University School of Medicine, Baltimore, Maryland, USA

${ }^{22}$ Northwestern University, Feinberg School of Medicine, Chicago, Illinois, USA

${ }^{23}$ Department of Medicine University of Puerto Rico Medical Sciences Campus, San Juan, Puerto Rico

${ }^{24}$ Gwen Knapp Center for Lupus and Immunology Research, University of Chicago, Chicago, Illinois, USA

${ }^{25}$ Department of Immunology, Instituto de Biomedicina y Parasitolog'a L-pez-Neyra, CSIC, Granada, Spain

${ }^{26}$ Department of Medicine, Sanatorio Parque, Rosario, Argentina

${ }^{27}$ King s College London, Divisions of Genetics and Molecular Medicine and Immunology, Infection and In ammatory Disease, Guy s Hospital, London, UK ${ }^{28}$ Departments of Biostatistical Sciences and Internal Medicine, Wake Forest School of Medicine, Winston-Salem, North Carolina, USA

${ }^{29}$ Division of Rheumatology, The University of Texas Health Science Center at Houston, Houston, Texas, USA

30 Pediatrics and US Department of Veterans Affairs Medical Center, Cincinnati

Children s Hospital Medical Center, Cincinnati, Ohio, USA

\section{REFERENCES}

1. Harley JB, Alarc-n-Riquelme ME, Criswell LA, et al. Genome-wide association scan in women with systemic lupus erythematosus identi es susceptibility variants in ITGAM, PXK, KIAA1542 and other loci. Nat Genet 2008;40:204 10.

2. Nath SK, Han S, Kim-Howard X, et al. A nonsynonymous functional variant in integrin-alpha(M) (encoded by ITGAM) is associated with systemic lupus erythematosus. Nat Genet 2008;40:152 4.

3. Hochberg MC. Updating the American College of Rheumatology revised criteria for the classi cation of systemic lupus erythematosus. Arthritis Rheum 1997;40:1725.

4. Adrianto I, Wen F, Templeton A, et al. Association of a functional variant downstream of TNFAIP3 with systemic lupus erythematosus. Nat Genet 2011;43:253 8.

5. Purcell S, Neale B, Todd-Brown K, et al. PLINK: a tool set for wholegenome association and population-based linkage analyses. Am J Hum Genet 2007:81:559 75

6. Huedo-Medina TB, S nchez-Meca J, Mar'n-Mart'nez F, et al. Assessing heterogeneity in meta-analysis: 0 statistic or 12 index? Psychol Methods 2006;11:193 206.

7. Mägi R, Morris AP. GWAMA: software for genome-wide association meta-analysis. BMC Bioinformatics 2010;11:288.

8. Cordell HJ. Detecting gene-gene interactions that underlie human diseases. Nat Rev Genet 2009;10:392 404 .

9. Dimas AS, Deutsch S, Stranger BE, et al. Common regulatory variation impacts gene expression in a cell type-dependent manner. Science 2009;325:1246 50.

10. Yang TP, Beazley C, Montgomery SB, et al. Genevar: a database and Java application for the analysis and visualization of SNP-gene associations in eQTL studies. Bioinformatics 2010;26:2474 6.

11. Han S, Kim-Howard X, Deshmukh $\mathrm{H}$, et al. Evaluation of imputation-based association in and around the integrin-alpha-M (ITGAM) gene and replication of robust association between a non-synonymous functional variant within ITGAM and systemic lupus erythematosus (SLE). Hum Mol Genet 2009;18:1171 80.
12. van de Stolpe A, van der Saag PT. Intercellular adhesion molecule-1. J Mol Med 1996;74:13 33

13. Gahmberg CG, Tian L, Ning L, et al. ICAM-5 a novel two-facetted adhesion molecule in the mammalian brain. Immunol Lett 2008;117:131 5.

14. Toivanen A, Ihanus $\mathrm{E}$, Mattila $\mathrm{M}$, et al. Importance of molecular studies on major blood groups intercellular adhesion molecule-4, a blood group antigen involved in multiple cellular interactions. Biochim Biophys Acta 2008;1780:456 66.

15. Paré G, Chasman DI, Kellogg M, et al. Novel association of $A B O$ histo-blood group antigen with soluble ICAM-1: results of a genome-wide association study of 6,578 women. PLoS Genet 2008;4:e1000118.

16. Barbalic M, Dupuis J, Dehghan A, et al. Large-scale genomic studies reveal central role of $\mathrm{ABO}$ in sP-selectin and sICAM-1 levels. Hum Mol Genet 2010;19:1863 72

17. Egerer K, Feist E, Rohr U, et al. Increased serum soluble CD14, ICAM-1 and E-selectin correlate with disease activity and prognosis in systemic lupus erythematosus. Lupus 2000;9:614 21

18. Sabry A, Sheashaa H, El-Husseini A, et al. Intercellular adhesion molecules in systemic lupus erythematosus patients with lupus nephritis. Clin Rheumatol 2007;26:1819 23.

19. Sfikakis PP, Tsokos GC. Clinical use of the measurement of soluble cell adhesion molecules in patients with autoimmune rheumatic diseases. Clin Diagn Lab Immunol 1997:4:241 6

20. Tso TK, Huang WN. Elevated soluble intercellular adhesion molecule-1 levels in patients with systemic lupus erythematosus: relation to insulin resistance. J Rheumatol 2007;34:726 30

21. Tulek N, Aydintug 0, Ozoran K, et al. Soluble intercellular adhesion molecule-1 (sICAM-1) in patients with systemic lupus erythematosus. Clin Rheumatol 1996;15:47 50.

22. Anaya JM, Kim-Howard X, Prahalad S, et al. Evaluation of genetic association between an ITGAM non-synonymous SNP (rs1143679) and multiple autoimmune diseases. Autoimmun Rev 2012;11:276 80

23. Abbas AR, Baldwin $\mathrm{D}, \mathrm{Ma} Y$, et al. Immune response in silico (IRIS): immune-speci c genes identi ed from a compendium of microarray expression data. Genes Immun 2005;6:319 31.

24. Hynes RO. Integrins: versatility, modulation, and signaling in cell adhesion Cell 1992;69:11 25

25. Lu H, Smith CW, Perrard J, et al. LFA-1 is suf cient in mediating neutrophil emigration in Mac-1-de cient mice. J Clin Invest 1997;99:1340 50.

26. Fagerholm SC, Varis M, Stefanidakis $M$, et al. alpha-Chain phosphorylation of the human leukocyte CD11b/CD18 (Mac-1) integrin is pivotal for integrin activation to bind ICAMs and leukocyte extravasation. Blood 2006;108:3379 86.

27. Li Y, Zhang L. The fourth blade within the beta-propeller is involved specifically in C3bi recognition by integrin alpha M beta 2. J Biol Chem 2003;278:34395 402.

28. Sachs UJ, Chavakis T, Fung $\mathrm{L}$, et al. Human alloantibody anti-Mart interferes with Mac-1-dependent leukocyte adhesion. Blood 2004;104:727 34

29. Buyon JP, Shadick N, Berkman R, et al. Surface expression of Gp 165/95, the complement receptor CR3, as a marker of disease activity in systemic Lupus erythematosus. Clin Immunol Immunopathol 1988;46:141 9

30. Hepburn AL, Mason JC, Wang S, et al. Both Fcgamma and complement receptors mediate transfer of immune complexes from erythrocytes to human macrophages under physiological ow conditions in vitro. Clin Exp Immunol 2006:146:133 45. 